\title{
Working with Individuals
}

Peter McGill, The Tizard Centre, University of Kent

Some years ago, when starting a new course on working with people with challenging behaviour, I cast around for published accounts of work with individuals that I could show to students as models for their own assignments. While I eventually found some useful articles, the search proved much more difficult than I had anticipated. Many of the articles I found had to be ruled out for one or another reason. Some were (however scientifically valuable) far too technical to be of use to most practitioners. Some used methods (e.g., punishment) that were no longer generally acceptable. Some (however interesting) paid no attention to evaluating the effectiveness of the work reported.

Since this experience I have been on the look-out for good quality accounts of work with individuals that would be of benefit to other practitioners. The British Journal of Learning Disabilities has published a few over the years and I was very pleased to get the opportunity to edit a Special Issue devoted to such accounts. In the invitation to authors I identified a number of criteria for articles to be submitted and will repeat these here as they define (at least for me) the most important characteristics of good quality work. It was hoped that all articles would include clear descriptions of the following:

\section{The person with learning disabilities and their life circumstances}

This is important for at least two reasons. Firstly, it helps readers to judge the relevance of the report to other individuals e.g., those that they, themselves, are working with. Clearly, some approaches to supporting individuals are likely only to be useful with individuals with certain characteristics. Some approaches might be particularly suited to people with good verbal 
abilities, others might require that the person has access to particular sorts of experiences or opportunities on a regular basis. Secondly, accounts that do not introduce the person and their life circumstances would seem to ignore one of the most important lessons of the past 20 years - we are not working with "behaviours" or "skills" or other disembodied elements, we are working with people who have lives outside of our contact with them and whose behaviour, skills and other characteristics can only be properly understood by a knowledge of the contexts (personal, historical, environmental and so on) of their lives. The papers in this Issue are, therefore, offered not as clinical case studies but as accounts of work with people.

\section{The methods of support and intervention}

If one mistake is to conceptualise our work with individuals in an overly clinical fashion, surely another is to forget that the process of supporting a person with learning disabilities may require technical knowledge, skills and methods of a high order. Authors were, therefore, asked to describe the methods they used so that the links between what they did and the outcomes for the individual would be clear and readers would have the opportunity to follow up on methods of relevance to their own work. We should not be afraid of "technology". Just as we expect doctors and others to both treat us as people (not hips or livers or gall bladders) and also be technically skilled at their jobs so people with learning disabilities are entitled to similar expectations of those that support them.

\section{The methods used for evaluation and the data arising from evaluation}

The notion of evidence-based practice has become common (in discussion if not in practice!). The notion is greatly needed in our work. A person with challenging behaviour is referred to a peripatetic support team. What determines whether they receive behavioural assessment and intervention, individual 1-1 therapy, a dietary intervention, gentle teaching or medication? Of 
course, practitioners would rightly note that characteristics of the person influence the support they receive but it is undoubtedly the case that much of the variation in this support is determined by other factors, such as the professional background of the person whom (by chance) they see, the local availability of certain sorts of support, the local or national "fashion" for particular sorts of interventions and so on. This is not to criticise practitioners, who do the best they can, but to draw attention to the extent to which practice with individuals is often not evidence-based. Accordingly, the articles in this Issue present information both about the outcomes of the work done, and about the methods used to gather this information, so that readers can judge the extent to which the conclusions reached by authors are valid. All of the articles in this Issue could be criticised for being poor science. Inevitably, however, there is a trade-off between the demands of everyday practice and the rigour of experimental design. Good quality practice requires at least an acknowledgement of this trade-off and the drawing of suitably modest conclusions about the impact of the work reported.

Before concluding I would like to note two regrets. Firstly, I had hoped that more articles would be submitted for this Issue. There is clearly a great deal of work being done with individuals throughout the country. We may need to find ways beyond traditional journals to encourage the dissemination and sharing of this work as this is an important way of spreading the use of best practice. Second, all of the articles in this Issue, however positive the approaches taken, have the reduction or management of challenging behaviour as their context. I am sure that this reflects the priorities of practitioners working under pressure. It would be nice, however, if any future such Issues also contain accounts of constructive work to promote development, employment, relationships, participation (and so on) that are not prompted by concerns about challenging behaviour. 
I hope that readers find the articles in this Issue useful in their work. I know that I will be using them in my own teaching! I enjoyed editing this Issue and would like to thank all the authors who submitted articles, and those colleagues who (often to short timelines) reviewed articles for me. I am also grateful to John Harris, Berni Moorcroft and Jan Walmsley for their invaluable advice. 